\title{
An empirical analysis of the impact of income inequality and social capital on physical and mental health - take China's micro-database analysis as an example
}

\author{
Yuanyuan $\mathrm{He}^{1}$, Lulin Zhou ${ }^{1 *}$, Junshan $\mathrm{Li}^{2}$ and Jun $\mathrm{Wu}^{2}$
}

\begin{abstract}
Background: Income inequality is one of the important reflections of the unbalanced development of the world economy and can have adverse effects on physical and mental health.

Methods: This article used the 2018 China Family Panel Studies Database as an empirical analysis data source. The Kakwani index (KI) was used to measure income inequality, and social capital was broken into cognitive social capital and structural social capital. Our assessment was conducted by using STATA16 software for ordered logistic regression, verifying income inequality, social capital on correlation between physical and mental health firstly; then by gradual regression methods to verify intermediary effect, and demonstrate the social capital as an intermediary variable affecting physical and mental health as income inequality.

Result: The income inequality has a significant negative effect on physical and mental health $(\beta=-0.964,-0.381$; $\mathrm{OR}=0.382,0.758 ; P<0.01)$, Social capital has a significant effect on physical and mental health (Cognitive SC(MH): $\beta=0.146$ and $0.104, O R=1.157$ and $1.110, P<0.01$; Cognitive $S C(P H): \beta=0.046$ and $0.069, O R=1.047$ and 1.071 , $P<0.01$; Structural SC(MH): $\beta=-0.005,0.025$ and $0.015, \mathrm{OR}=0.995,1.025$ and $1.015, P>0.1, P<0.01$ and $P<0.01$; Structural SC(PH): $\beta=-0.026,0.009$ and $-0.013, \mathrm{OR}=0.975,1.009$ and $0.987, P<0.01, P>0.1$ and $P<0.01)$. Our analysis also showed that social capital (cognitive social capital and structural social capital) has an intermediary effect on physical and mental health due to income inequality.

Conclusion: This study shows that income inequality can not only directly affect physical and mental health, but also through social capital intermediary utility indirectly affect physical and mental health, social capital has positive effects on physical and mental health. At the same time, income inequality and social capital's effects on physical and mental health exist regional differences, urban-rural differences, and gender differences. Therefore, in the development of special policies to support and take care of vulnerable groups, special attention needs to be paid to poor rural areas and female groups.
\end{abstract}

Keywords: Income inequality, Kakwani index, Social capital, Physical and mental health, Intermediary effect

*Correspondence: 525939756@qq.com

1 School of Management, Jiangsu University, Zhenjiang 212002, Jiangsu, China

Full list of author information is available at the end of the article

\section{Introduction}

According to the 2020 World Social Report of the Department of Economic and Social Affairs of the United Nations, issues of inequality remain endemic in both developed- and developing- countries. The World original author(s) and the source, provide a link to the Creative Commons licence, and indicate if changes were made. The images or other third party material in this article are included in the article's Creative Commons licence, unless indicated otherwise in a credit line to the material. If material is not included in the article's Creative Commons licence and your intended use is not permitted by statutory regulation or exceeds the permitted use, you will need to obtain permission directly from the copyright holder. To view a copy of this licence, visit http://creativecommons.org/licenses/by/4.0/. The Creative Commons Public Domain Dedication waiver (http://creativeco mmons.org/publicdomain/zero/1.0/) applies to the data made available in this article, unless otherwise stated in a credit line to the data. 
Health Organization estimates that about $70 \%$ of the world's population still lives in inequality-exacerbated countries, and the level of inequality is in a historic high today. Among them, income inequality (Ilequality) is the main problem, which is the reflection of unbalanced economic development [1, 2]. Although the Ilequality between countries has been reduced, this issue is getting increasingly serious, especially in developing countries [3]. China is no exception and IIequality is one of the main reasons why health revenue stagnation, resulting in decreased human life by 0.4 years for women and 0.6 years for men [4].

The path that IIequality effects on physical and mental health $(\mathrm{PH}$ and $\mathrm{MH})$ is divided into direct path and indirect two ways [5]. The analysis of the impact mechanism of income affecting health mainly revolves around the dimensions of absolute income, relative income and income deprivation [6]. First, the Ilequality can directly act on individual $\mathrm{PH}$ and $\mathrm{MH}$, resulting in increased mortality [7], shortening per capita life [8], affecting the physiological functions [9] and $\mathrm{MH}$ [10]. Second, Ilequality can be indirectly active in $\mathrm{PH}$ and $\mathrm{MH}$ through eroding social capital (SC) [11]. Although more and more evidence suggests that IIequality is related to $\mathrm{PH}$ and $\mathrm{MH}$, there is still disagreement in the research conclusions of IIequality and $\mathrm{PH}$ and $\mathrm{MH}$ [12]. A more in-depth assessment is needed in the future. Especially, current researches on Ilequality are concentrated more in the group level $[13,14]$ and national level $[7,15]$, but relatively few at a personal level.

Social capital can affect both the IIequality and $\mathrm{PH} \& \mathrm{MH}$ of an individual. Regarding, IIequality, SC plays a double role. Firstly, it can enhance personal endowment, increase income, improve social status through its poverty reduction effect and stabilization effect, and improve IIequality [16]. On the other hand, SC can weaken capital liquidity by influencing investment behavior and capital transmission paths, leading to continued widening of the income gap [17]. Secondly, SC has an impact on $\mathrm{PH}$ and $\mathrm{MH}$. SC can directly affect $\mathrm{PH}$ and $\mathrm{MH}$ through social equity, social trust, and social network density which can in turn positively impact on an individual's self-rated health and self-rated $\mathrm{MH}$. Further, the extant literature also presents a plethora of studies that support that SC can significantly reduce mortality, suicide rate, and alleviate depression [18-20]. Moreover, SC can affect a person's health by affecting medical services utilization. In the work of Zhao et al. (2019), it is observed that different types of SC can have different effects on health [21, 22].
Even though more and more scholars are concerned about the impact of $\mathrm{SC}$ on Ilequality and $\mathrm{PH}$ and $\mathrm{MH}$, there are few studies on the synergy of the three elements [23, 24]. Specifically, there is a paucity of studies that systematically demonstrate the intermediary role of SC in the relationship between Ilequality and health ( $\mathrm{PH}$ and $\mathrm{MH}$ ).

Regression analysis has been the common method currently used to assess the relationship between IIequality, SC and $\mathrm{PH}$ and $\mathrm{MH}$ [25], with more focus on adolescents [24] and the elderly [11] in developed countries. There is a lack of relevant arguments for developing countries. This study selected China as the research object, which is the largest developing country. The data source is the results of China Family Panel Studies (CFPS) in 2018. This is advantageous to validating the interplay between IIequality, SC and health because 2018 CFPS is the single largest source of data pertaining to the SC, health, and Ilequality and other related measurement indicators for one country. The standard estimate of "household income per capita" and "individual income" for 2018 as revealed by the CFPS indicates that China's Gini coefficients are 0.508 and 0.477 , respectively, which are both higher than the 0.4 warning level. This indicates that the current income gap in China is excessively serious, thus requiring immediate attention to resolve to avert further exacerbation. Navigating the reason why China's persistent and resilient economic development has not translated into total eradication of a large tract of economic disparity is of academic interest. The study then applies ordered logistic regression and other methods to do robustness tests to study the impact of IIequality and SC on PH and MH. Subsequently, the KHB (Karlson-Holm-Breen) method is applied to test the mediating effect of $\mathrm{SC}$ in the path of IIequality on $\mathrm{PH}$ and $\mathrm{MH}$. The findings of this study will have important enlightenment for developing countries to solve social problems caused by IIequality.

\section{Research design and method section Data source and processing}

The research data came from China's 2018 CFPS database, which is a national social survey project with a two-year survey cycle, including individual, family, community, and other levels of survey data. The survey covered 31 provinces/cities/autonomous regions of China including 14 in Eastern China, 6 in Central China, and 11 in Western China covering nearly $95 \%$ of the regional population. It is a typical and representative micro-database in China with more than 30, 000 
interviewees. The study selected the content of family questionnaires and adult questionnaires as the main data sources $(N=33,326)$, and eliminated invalid data such as "null values, refusal to answer or don't know". The effective sample size is 22,751 , women accounted for $52.21 \%$ and men accounted for $47.79 \%$, rural population was $50.75 \%$ and urban population was $49.25 \%$, the average age of the sample was 47.76 , and the average years of education was 7.325 years. The proportion of participating in basic medical insurance is $91.6 \%$ (including medical insurance for urban employees and urban residents). Because the data source is cross-sectional, a multicollinearity test for verifying variance inflation factor and Tolerance were performed to avoid the linear correlation of the explanatory variables from affecting the regression results. Except for the "region" variable, the variance inflation factor value of other analysis variables are all less than 2 , and Tolerance values are all more than 0.5 .

\section{Method section and model construction}

Because the assignments of variables related to $\mathrm{PH}$ and $\mathrm{MH}$ are ordinal categorical variables, the ordered logistic regression model was used for model estimation. The measurement model is as follows:

$$
\text { Health }_{i}^{*}=\beta X_{i}+\operatorname{prov}_{i}+\mu_{i}+\varepsilon_{i}(i=1,2, \sim n)
$$

The variable $\mathrm{Health}_{i}$, refers $\mathrm{PH}$ and $\mathrm{MH}$, they are unobservable latent variables; IIequality, $\mathrm{SC}$ and other $\mathrm{CVs}$ are all included in the vector $X_{i} ; \mu_{\mathrm{i}}$ is the individual effect; and $\operatorname{prov}_{i}$ refers to province fixed effect; $\varepsilon_{i}$ is a random error term that obeys a logical distribution, the individual selection rule is:

$$
\text { Health }_{i}^{*}=\left\{\begin{array}{l}
1, \text { if }\left(\text { Health }_{i}^{*} \leq r_{0}\right) \\
2, \text { if } \quad\left(r_{0} \leq\right. \text { Health } \\
3, \text { if } \quad\left(r_{1} \leq r_{1}\right) \\
4, \text { if } \quad\left(r_{2} \leq \text { Health }{ }_{i}^{*} \leq r_{2}\right) \\
5, \text { if } \quad\left(r_{3} \leq \text { Health }_{i}^{*} \leq r_{4}\right)
\end{array}\right.
$$

Among them, $r_{0}<r_{1}<r_{2}<r_{3}<r_{4}$, they are called the cutoff points, $\varepsilon_{i}$ obeys the logical distribution, $\mathrm{F}(\phi)=\exp$. $(\phi) /[1+\exp .(\phi)]$, so the probability distribution function is expressed as:

$$
\begin{aligned}
P & =\left(\text { Health }_{i}=2 \mid X_{i}, \beta, \operatorname{prov}_{i}, \mu_{i}\right) \\
& =F\left(r_{1}-X_{i} \beta-\mu_{i}-\operatorname{prov}_{i}\right) \\
& -F\left(r_{0}-X_{i} \beta-\mu_{i}-\operatorname{prov}_{i}\right) \\
P & =\left(\text { Health }_{i}=3 \mid X_{i}, \beta, \operatorname{prov}_{i}, \mu_{i}\right) \\
& =F\left(r_{2}-X_{i} \beta-\mu_{i}-\operatorname{prov}_{i}\right) \\
& -F\left(r_{1}-X_{i} \beta-\mu_{i}-\operatorname{prov}_{i}\right) \\
P & =\left(\text { Health }_{i}=4 \mid X_{i}, \beta, \operatorname{prov}_{i}, \mu_{i}\right) \\
& =1-F\left(r_{3}-X_{i} \beta-\mu_{i}-\operatorname{prov}_{i}\right)
\end{aligned}
$$

Ilequality may erode $\mathrm{SC}$ to a certain extent. To further assess the mediating role of $\mathrm{SC}$ in the path of Ilequality affecting $\mathrm{PH}$ and $\mathrm{MH}$, the study adopted the $\mathrm{KHB}$ approach [26-28] to test the mediating utility of SC and constructed a mediating effect regression model. The KHB analysis method is suitable for estimating the intermediary variables of the nonlinear probability models. It can not only analyze the binary logistic regression model but also can be used to analyze the ordered multi-class logistic regression. The equation of the model is shown in the formulas 7, 8, 9 below:

$$
\begin{aligned}
& \text { Health }_{i}^{*}=\beta_{0}+\text { alInquality }_{i}+\gamma X_{i}+\text { prov }_{i}+\mu_{i}+\varepsilon_{i} \\
& S C_{i}=\beta_{1}+\text { bIInquality }_{i}+\gamma X_{i}+\text { prov }_{i}+\mu_{i}+\varepsilon_{i} \\
& \text { Health }_{i}^{*}=\beta_{2}+a^{\prime} \text { IInquality }_{i}+c S C_{i}+\gamma X_{i}+\mu_{i}+\text { prov }_{i}+\varepsilon_{i}
\end{aligned}
$$

Formula (7) tests the total effect of IIequality on health which is a "simplified model", formula (8) tests the correlation between Ilequality and SC, and formula (9) tests the correlation coefficients of independent variables after controlling SC which is a "full model"; Health $h_{i}$ are unobservable latent variables whose formulas are represented as (3)-(6), the Direct effect (DE), and Total effect (TE) are shown in the formula (10). Among them, $\sigma_{b}$ and $\sigma_{a}$ are the scale parameters, which are derived from the standard error of the residuals of the linear regression Eqs. (7) and (9), and $\sigma_{a} \leq \sigma_{b}$. Therefore, the measurement method of

$$
\begin{aligned}
& P=\left(\text { Health }_{i}=1 \mid X_{i}, \beta, \text { prov }_{i}, \mu_{i}\right)=P\left(\text { Health }_{i}^{*} \leq r_{0} \mid X_{i}, \beta, \text { prov }_{i}, \mu_{i}\right)=P\left(\beta X_{i}+\operatorname{prov}_{i}+\mu_{i}+\varepsilon_{i} \leq r_{0} \mid X_{i}, \beta, \mu_{i}\right) \\
& =P\left(\varepsilon_{i} \leq r_{0}-X_{i} \beta-\mu_{i}-\operatorname{prov}_{i} \mid X_{i}, \beta, \mu_{i}\right)=F\left(r_{0}-X_{i} \beta-\mu_{i}-\text { prov }_{i}\right)
\end{aligned}
$$


the Intermediary effect (IE) in the logistic model is shown in Eq. (11). The specific measurement results can be completed by STATA16 software.

$$
\begin{aligned}
& D E=\frac{a}{\sigma_{a}}, T E=\frac{b}{\sigma_{b}} \\
& I E=T E-D E=\frac{b}{\sigma_{b}}-\frac{a}{\sigma_{a}}
\end{aligned}
$$

\section{Variable selection \\ Dependent variable (DV)}

The health variables of regression analysis include two dimensions: $\mathrm{PH}$ and $\mathrm{MH}$. Physical health refers to the development cycle and severity of the disease as a whole and adopts "whether the body feels unwell in the past two weeks", "whether there is any chronic disease within six months", "have you seen a doctor", and "whether the cause is caused in the past 12 months". The above four indicators mainly measure individual $\mathrm{PH}$ by illness and hospitalization, if the answer of the indicator is "yes", it is assigned " 0 ", if the answer is "no", it is assigned " 1 ". According to the total score, four points of PH level 1-4 are obtained. Mental health is based on the eight psychological survey questions QN406-QN420 in the "Behavior and Mental State" module of the questionnaire, which mainly includes indicators such as personal sleep status and loneliness. According to the directional content of the indicators, conduct 1-4 Corresponding points. The total score of $\mathrm{MH}$ status is $8-32$ points.

\section{Independent variable (IV)}

The dependent variable includes two variables, namely IIequality and SC.

Income inequality (IIequality) The study used the individual income deprivation index (Kakwani index, KI) as a measure of IIequality at the individual level $[5,29]$, which emphasizes that Ilequality at the individual level comes from the comparison of individuals with high-income reference groups. Commonly used individual deprivation indexes are Yitzhaki index [30], KI [31] and Podder index [32]. The KI is the development of the Yitzhaki index, which overcomes the latter's sensitivity to changes in income scale [33], and at the same time makes up for the shortcomings of indecomposability and non-addition of the Gini coefficient. The relative deprivation of group members due to income gap can be observed objectively, and the Ilequality at the individual level can be visualized. Therefore, this study used the KI to measure IIequality and the specific calculation formula is as follows:

$$
R D\left(I, I_{i}\right)=\frac{1}{n \mu_{I}}\left[\sum_{j=i+1}^{n}\left(I_{j}-I_{i}\right)\right]=\gamma_{I_{i}}^{+}\left[\frac{\left(\mu_{I_{i}}^{+}-I_{i}\right)}{\mu_{I}}\right]
$$

Of which, household per capita income was used to calculate the individual income deprivation index. I is the reference income group composed of all the variables filtered in the CFPS database, the number of groups is $\mathrm{n}$, and the income of individuals in the reference group is arranged in ascending order to form an income vector set $\left(I_{1}, I_{2}, I_{3}, \ldots, I_{n}\right)$. In formula (12), $\mu_{\mathrm{I}}$ represents the average income of all samples in group $\mathrm{I}, \gamma_{I_{i}}^{+}$is the proportion of the number of samples in I group whose income exceeds Ii in the total sample volume of heatstroke, and $\mu_{I_{i}}^{+}$is the average of sample incomes in group I with income exceeding Ii. In the heterogeneity test, due to changes in the characteristics of the reference group, the vector set of the reference group will also change accordingly. The corresponding reference group income deprivation index was recalculated using the same method described above.

Social capital (SC) Social capital is a characteristic of social organizations, and it is the product of the fusion of subjective social norms and objective social characteristics. Among them, the organizational network and the mutual trust and reciprocity among members of the organization can strengthen the cooperative relationship between individual members, thereby promoting the efficiency of the organization and society. Improve [34]. Social capital is divided into cognitive and structural SC [35]. Cognitive SC refers to the mutual trust and mutual assistance between members of social organizations, which are measured by indicators of social justice and social trust. Structural SC refers to the network of relationships among members of an organization, which is measured basing on social support, relationship, and network density. First of all, the sense of social justice used N601 "How do you think the following problems are in our country" to comprehensively calculate scores, including housing, medical care, social security, wealth gap and other social issues. Secondly, the social trust was calculated by using a comprehensive score for the trust of parents, relatives, neighbors, strangers and government officials; Social support used the sum of social funds given by children, relatives and others and aid funds given to others by oneself as a measurement indicator; Personal relationship is closely related to the social resources that individuals can mobilize [36], so structural SC was included for targeted analysis, and QM2011 "How good is your relationship" question was used as the research indicator. Finally, China is a traditional relational society, 
and etiquette, obedience, and human relationship are important ways to maintain social networks and interpersonal relationships [37], so the FU201 "Relationship and Gift Expenditure Measurement" was used to measure the density of the relationship network $[38,39]$.

\section{Covariates}

Considering the impact of other factors on $\mathrm{PH}$ and $\mathrm{MH}$ and minimizing indigenousness and heteroscedasticity, this study incorporated the individual's characteristics (ICV), social characteristics (SCV), living habits, and medical environment (ME) into the analysis framework: a) Taking household registration, gender, age, marital status, years of education, region as ICVs; b) Working status, insurance participation, family size, social status, etc. were selected as SCVs; c) Smoking, drinking, exercise, lunch break, etc. were selected as descriptive variables for indications of lifestyle habits; and d) the patient's satisfaction with the diagnosis and treatment environment and the diagnosis and treatment level were considered as the ME. The basic description and statistical results of the variables are shown in Table 1.

\section{Robustness test}

This study conducted a variety of methods for robustness testing to ensure the science of research methods and research indicators and the reliability of research conclusions. At first, replace the index of the DV was replaced by taking self-rated health data as the explained variable and estimated the Ordered Logistic Regression according to the variable type as the ordered multicategory discrete variable. The test results are shown in Table 4 (1). Secondly, the IV form and the KI with Podder index were replaced by taking the logarithm of the family's per capita income and then calculate it according to the Yitzhaki index measurement method. The test results are shown in Table 4 columns (2) and (3). At the same time, in order to eliminate the influence of outliers on the regression results, the Winsor shrinking method was used to process the samples up and down $1 \%$ of the statistical indicators, and the remaining 22,296 samples were re-regressed. Finally, the test model was changed to convert the $\mathrm{MH}$ and $\mathrm{PH}$ data into continuous numerical variables. The total score of $\mathrm{MH}$ status is 8-32 points, and four points of $\mathrm{PH}$ are obtained, then the ordinary least squares regression was used for statistical analysis.

Table 1 Variable description and statistics

\begin{tabular}{|c|c|c|c|c|}
\hline Type & Name & Description & Mean & SD \\
\hline \multirow[t]{2}{*}{ Health Variable } & XIhealth & $\mathrm{MH}$, score range: $1-5$, the large score, the healthier & 4.239 & 0.818 \\
\hline & Kghealth & $\mathrm{PH}$, score range: $1-4$, the large score, the healthier & 3.378 & 0.845 \\
\hline Ilequality & Kakwani & calculated using $\mathrm{Kl}$, value is $0-1$ & 0.508 & 0.246 \\
\hline \multirow[t]{5}{*}{ SC } & Trust & Average value of trust, ranges $0-10$, large value means higher trust & 5.575 & 1.343 \\
\hline & Fair & Average value of fairness, score ranges $1-8$, fairness score from low to high & 3.309 & 1.540 \\
\hline & Support & Social support, total reciprocal financial assistance, logarithm & 4.220 & 3.983 \\
\hline & Relations & Relationship level: 0-10 level increasing & 7.109 & 1.981 \\
\hline & Jiegousc & Network density, measured by expense of favors gifts, logarithm & 7.277 & 2.437 \\
\hline \multirow[t]{5}{*}{ ICV } & Urban & Household registration, urban $=1$, rural $=0$ & 0.493 & 0.500 \\
\hline & Gender & Gender: male $=1$, female $=0$ & 0.478 & 0.500 \\
\hline & Age & Age, actual age, value ranges: $20-81$ & 47.77 & 15.44 \\
\hline & Marriage & Marital status, with spouse $=1$, without spouse $=0$ & 0.825 & 0.380 \\
\hline & Education & Years of education, from illiterate to doctorate, value range: 0-22 & 7.331 & 4.920 \\
\hline \multirow[t]{4}{*}{ SCV } & Employ & Working status, employed $=1$, unemployed $=0$ & 0.777 & 0.416 \\
\hline & Insurance & Insurance participation, Is there social medical insurance? Yes $=1, \mathrm{No}=0$ & 0.916 & 0.277 \\
\hline & Family & Family size, number of family members, value range: 1-21 & 4.145 & 2.081 \\
\hline & Status & Social status, range: $1-5$, mean from low to high & 3.103 & 1.092 \\
\hline \multirow[t]{4}{*}{ Living habit } & Exercise & Exercise, the frequency of physical exercise in last week. Value is 0-50 & 2.496 & 3.295 \\
\hline & Smoke & Smoking: Smoking in the past month? $\mathrm{Yes}=1, \mathrm{No}=0$ & 0.299 & 0.458 \\
\hline & Drink & Drinking: 3 times per week in the past month? $\mathrm{Yes}=1, \mathrm{No}=0$ & 0.155 & 0.362 \\
\hline & Break & Lunch break, Yes $=1, \mathrm{No}=0$ & 0.536 & 0.499 \\
\hline \multirow[t]{2}{*}{ ME } & Satisfaction & Medical condition, score range: $1-5$, means dissatisfied to satisfied & 3.628 & 0.814 \\
\hline & Care & Medical level, score range: $1-5$, from very bad to very good & 3.499 & 0.885 \\
\hline
\end{tabular}

SD Standard deviation, Ilequality Income inequality, SC Social capital, ICV Individual characteristic variables, SCV Social characteristic variables, ME Medical Environment, $M H$ Mental health, PH Physical health, KI Kakwani index 


\section{Heterogeneity validation}

In the analysis of the influence of the Covariates on the DV, it is found that both household registration and gender will have a significant impact on $\mathrm{PH}$ and $\mathrm{MH}$. It is speculated that there may also be gender differences and household registration differences in the effect of Ilequality and SC on the DV, therefore, gender and household registration are selected as the dimensions of the heterogeneity validation. In addition, the eastern, central, and western parts of China show a gradual decline in income levels, so regional variables are also used as one of the dimensions of heterogeneity validation.

Table 2 Regression results of the impact of llequality and SC on $\mathrm{MH}$

\begin{tabular}{|c|c|c|c|c|c|c|c|c|}
\hline \multirow[t]{2}{*}{ Variable } & & (1) & (2) & (3) & (4) & (5) & (6) & (7) \\
\hline & & \multicolumn{7}{|l|}{$\mathrm{MH}$} \\
\hline Ilequlity & KI & $\begin{array}{l}-0.962^{* * *} \\
(-14.55)\end{array}$ & & & & & & $\begin{array}{l}-0.964^{* * *} \\
(-14.19)\end{array}$ \\
\hline \multirow[t]{5}{*}{ SC } & Trust & & $\begin{array}{l}0.150^{* * *} \\
(-13.590)\end{array}$ & & & & & $\begin{array}{l}0.146^{* * *} \\
(-13.130)\end{array}$ \\
\hline & Fair & & & $\begin{array}{l}0.0977^{* * *} \\
(-10.820)\end{array}$ & & & & $\begin{array}{l}0.104^{* * *} \\
(-11.540)\end{array}$ \\
\hline & Support & & & & $0.002(-0.720)$ & & & $-0.005(-1.39)$ \\
\hline & Relations & & & & & $\begin{array}{l}0.028^{* * *} \\
(-4.120)\end{array}$ & & $\begin{array}{l}0.025^{* * *} \\
(-3.640)\end{array}$ \\
\hline & Jiegousc & & & & & & $\begin{array}{l}0.029^{* * * *} \\
(-5.250)\end{array}$ & $\begin{array}{l}0.015^{* * *} \\
(-2.580)\end{array}$ \\
\hline \multirow[t]{5}{*}{ IVC } & Urban & $\begin{array}{l}0.0809^{* * *} \\
(-2.810)\end{array}$ & $\begin{array}{l}0.169^{* * *} \\
(-6.010)\end{array}$ & $\begin{array}{l}0.187^{* * *} \\
(-6.650)\end{array}$ & $\begin{array}{l}0.183^{* * *} \\
(-6.480)\end{array}$ & $\begin{array}{l}0.170^{* * *} \\
(-6.050)\end{array}$ & $\begin{array}{l}0.169^{* * *} \\
(-6.010)\end{array}$ & $\begin{array}{l}0.113^{* * *} \\
(-3.910)\end{array}$ \\
\hline & Gender & $\begin{array}{l}0.490^{* * *} \\
(-14.080)\end{array}$ & $\begin{array}{l}0.462^{* * *} \\
(-13.320)\end{array}$ & $\begin{array}{l}0.456^{* * *} \\
(-13.140)\end{array}$ & $\begin{array}{l}0.453^{* * *} \\
(-13.050)\end{array}$ & $\begin{array}{l}0.463^{* * *} \\
(-13.340)\end{array}$ & $\begin{array}{l}0.469^{* * *} \\
(-13.510)\end{array}$ & $\begin{array}{l}0.480^{* * *} \\
(-13.740)\end{array}$ \\
\hline & Age & $\begin{array}{l}-0.002^{*} \\
(-1.83)\end{array}$ & $\begin{array}{l}-0.003^{* *} \\
(-2.32)\end{array}$ & $\begin{array}{l}-0.003^{* * *} \\
(-3.12)\end{array}$ & $\begin{array}{l}-0.004^{* * *} \\
(-3.57)\end{array}$ & $\begin{array}{l}-0.003^{* *} \\
(-2.30)\end{array}$ & $\begin{array}{l}-0.002^{* *} \\
(-2.04)\end{array}$ & $\begin{array}{l}-0.004^{* * *} \\
(-3.78)\end{array}$ \\
\hline & Marriage & $\begin{array}{l}0.261^{* * *} \\
(-7.080)\end{array}$ & $\begin{array}{l}0.278^{* * *} \\
(-7.570)\end{array}$ & $\begin{array}{l}0.295^{* * *} \\
(-8.030)\end{array}$ & $\begin{array}{l}0.290^{* * *} \\
(-7.890)\end{array}$ & $\begin{array}{l}0.278^{* * *} \\
(-7.580)\end{array}$ & $\begin{array}{l}0.265^{* * *} \\
(-7.200)\end{array}$ & $\begin{array}{l}0.285^{* * *} \\
(-7.700)\end{array}$ \\
\hline & Education & $\begin{array}{l}0.028^{* * *} \\
(-8.070)\end{array}$ & $\begin{array}{l}0.041^{* * *} \\
(-12.070)\end{array}$ & $\begin{array}{l}0.039^{* * *} \\
(-11.420)\end{array}$ & $\begin{array}{l}0.0461^{* * *} \\
(-13.410)\end{array}$ & $\begin{array}{l}0.0413^{* * *} \\
(-12.110)\end{array}$ & $\begin{array}{l}0.041^{* * *} \\
(-11.870)\end{array}$ & $\begin{array}{l}0.031^{* * *} \\
(-8.720)\end{array}$ \\
\hline \multirow[t]{4}{*}{ SCV } & Employ & $\begin{array}{l}0.066^{*} \\
(-1.950)\end{array}$ & $\begin{array}{l}0.071^{* *} \\
(-2.080)\end{array}$ & $\begin{array}{l}0.059^{*} \\
(-1.730)\end{array}$ & $\begin{array}{l}0.072^{* *} \\
(-2.100)\end{array}$ & $\begin{array}{l}0.071^{* *} \\
(-2.100)\end{array}$ & $\begin{array}{l}0.064^{*} \\
(-1.880)\end{array}$ & $0.053^{*}(-1.550)$ \\
\hline & Insurance & $\begin{array}{l}0.124^{* * *} \\
(-2.690)\end{array}$ & $\begin{array}{l}0.124^{* * *} \\
(-2.700)\end{array}$ & $\begin{array}{l}0.117^{* *} \\
(-2.540)\end{array}$ & $\begin{array}{l}0.127^{* * *} \\
(-2.760)\end{array}$ & $\begin{array}{l}0.120^{* * *} \\
(-2.610)\end{array}$ & $\begin{array}{l}0.119^{* * *} \\
(-2.580)\end{array}$ & $0.112^{* *}(-2.420)$ \\
\hline & Family & $\begin{array}{l}0.081^{* * *} \\
(-11.630)\end{array}$ & $\begin{array}{l}0.062^{* * *} \\
(-9.070)\end{array}$ & $\begin{array}{l}0.059^{* * *} \\
(-8.660)\end{array}$ & $\begin{array}{l}0.063^{* * *} \\
(-9.240)\end{array}$ & $\begin{array}{l}0.061^{* * *} \\
(-9.050)\end{array}$ & $\begin{array}{l}0.058^{* * *} \\
(-8.460)\end{array}$ & $\begin{array}{l}0.077^{* * *} \\
(-10.950)\end{array}$ \\
\hline & Status & $\begin{array}{l}0.067^{* * *} \\
(-5.600)\end{array}$ & $\begin{array}{l}0.0642^{* * *} \\
(-5.380)\end{array}$ & $\begin{array}{l}0.0571^{* * *} \\
(-4.770)\end{array}$ & $\begin{array}{l}0.0626^{* * *} \\
(-5.240)\end{array}$ & $\begin{array}{l}0.0523^{* * *} \\
(-4.260)\end{array}$ & $\begin{array}{l}0.0639^{* * *} \\
(-5.360)\end{array}$ & $\begin{array}{l}0.048^{* * *} \\
(-3.870)\end{array}$ \\
\hline \multirow[t]{4}{*}{ Living habit } & Exercise & $\begin{array}{l}0.0323^{* * *} \\
(-7.690)\end{array}$ & $\begin{array}{l}0.0346^{* * *} \\
(-8.230)\end{array}$ & $\begin{array}{l}0.0339^{* * *} \\
(-8.050)\end{array}$ & $\begin{array}{l}0.0354^{* * *} \\
(-8.380)\end{array}$ & $\begin{array}{l}0.0344^{* * *} \\
(-8.180)\end{array}$ & $\begin{array}{l}0.0344^{* * *} \\
(-8.200)\end{array}$ & $\begin{array}{l}0.0319^{* * *} \\
(-7.550)\end{array}$ \\
\hline & Smoke & $\begin{array}{l}-0.0984^{* * *} \\
(-2.69)\end{array}$ & $\begin{array}{l}-0.0945^{* * *} \\
(-2.59)\end{array}$ & $\begin{array}{l}-0.0823^{* *} \\
(-2.25)\end{array}$ & $\begin{array}{l}-0.0883^{* *} \\
(-2.42)\end{array}$ & $\begin{array}{l}-0.0955^{* * *} \\
(-2.62)\end{array}$ & $\begin{array}{l}-0.0985^{* * *} \\
(-2.70)\end{array}$ & $\begin{array}{l}-0.0825^{* *} \\
(-2.25)\end{array}$ \\
\hline & Drink & $\begin{array}{l}0.0704^{*} \\
(-1.810)\end{array}$ & $\begin{array}{l}0.0811^{* *} \\
(-2.100)\end{array}$ & $\begin{array}{l}0.0878^{* *} \\
(-2.270)\end{array}$ & $\begin{array}{l}0.0807^{* *} \\
(-2.090)\end{array}$ & $\begin{array}{l}0.0817^{* *} \\
(-2.110)\end{array}$ & $\begin{array}{l}0.0785^{* *} \\
(-2.030)\end{array}$ & $\begin{array}{l}0.0748^{*} \\
(-1.920)\end{array}$ \\
\hline & Break & $0.010(-0.360)$ & $0.017(-0.650)$ & $0.016(-0.610)$ & $0.019(-0.720)$ & $0.018(-0.660)$ & $0.015(-0.550)$ & $0.009(-0.350)$ \\
\hline \multirow[t]{2}{*}{ ME } & Satisfaction & $\begin{array}{l}0.114^{* * *} \\
(-5.690)\end{array}$ & $\begin{array}{l}0.109^{* * *} \\
(-5.480)\end{array}$ & $\begin{array}{l}0.0819^{* * *} \\
(-4.090)\end{array}$ & $\begin{array}{l}0.0973^{* * *} \\
(-4.880)\end{array}$ & $\begin{array}{l}0.110^{* * *} \\
(-5.50)\end{array}$ & $\begin{array}{l}0.111^{* * *} \\
(-5.560)\end{array}$ & $\begin{array}{l}0.076^{* * *} \\
(-3.770)\end{array}$ \\
\hline & Level & $\begin{array}{l}0.148^{* * *} \\
(-7.890)\end{array}$ & $\begin{array}{l}0.145^{* * *} \\
(-7.760)\end{array}$ & $\begin{array}{l}0.120^{* * *} \\
(-6.410)\end{array}$ & $\begin{array}{l}0.137^{* * *} \\
(-7.320)\end{array}$ & $\begin{array}{l}0.144^{* * *} \\
(-7.730)\end{array}$ & $\begin{array}{l}0.146^{* * *} \\
(-7.840)\end{array}$ & $\begin{array}{l}0.116^{* * *} \\
(-6.130)\end{array}$ \\
\hline Observations & & 22,751 & 22,751 & 22,751 & 22,751 & 22,751 & 22,751 & 22,751 \\
\hline Province & & YES & YES & YES & YES & YES & YES & YES \\
\hline Prob $>\mathrm{F}$ & & 0.000 & 0.000 & 0.000 & 0.000 & 0.000 & 0.000 & 0.000 \\
\hline Pseudo $R^{2}$ & & 0.040 & 0.036 & 0.040 & 0.038 & 0.036 & 0.036 & 0.047 \\
\hline
\end{tabular}




\section{Results}

Benchmark regression results

As shown in Tables 2 and 3, Benchmark regression included two main parts: the first is the independent regression of the IV that controls other key variables, and the second is the integrated regression that integrates all variables (column (7)).

\section{The impact of income inequality on physical and mental health}

The data in column (1) of Tables 2 and 3 both show that the coefficient of effect of Ilequality on $\mathrm{PH}$ and $\mathrm{MH}$ is significantly negative ( $\mathrm{MH}: \beta=-0.962, \mathrm{OR}=0.382$; PH: $\beta=-0.280, \mathrm{OR}=0.756 ; P<0.01)$. Integrating other variables (column (7)), the regression coefficient is still

Table 3 Regression results of the impact of llequality and SC on PH

\begin{tabular}{|c|c|c|c|c|c|c|c|c|}
\hline \multirow[t]{2}{*}{ Variable } & & (1) & $(2)$ & (3) & (4) & (5) & (6) & (7) \\
\hline & & \multicolumn{7}{|l|}{$\mathrm{PH}$} \\
\hline Ilequlity & KI & $\begin{array}{l}-0.280^{* * *} \\
(-4.08)\end{array}$ & & & & & & $\begin{array}{l}-0.381^{* * *} \\
(-5.44)\end{array}$ \\
\hline \multirow[t]{5}{*}{ SC } & Trust & & $\begin{array}{l}0.0471^{* * *} \\
(-4.200)\end{array}$ & & & & & $\begin{array}{l}0.0460^{* * *} \\
(-4.090)\end{array}$ \\
\hline & Fair & & & $\begin{array}{l}0.0674^{* * *} \\
(-7.210)\end{array}$ & & & & $\begin{array}{l}0.0687^{* * *} \\
(-7.330)\end{array}$ \\
\hline & Support & & & & $\begin{array}{l}-0.024^{* * *} \\
(-6.82)\end{array}$ & & & $\begin{array}{l}-0.026^{* * *} \\
(-7.26)\end{array}$ \\
\hline & Relations & & & & & $0.009(-1.280)$ & & $0.009(-1.320)$ \\
\hline & Jiegousc & & & & & & $\begin{array}{l}-0.010^{*} \\
(-1.77)\end{array}$ & $\begin{array}{l}-0.013^{* *} \\
(-2.21)\end{array}$ \\
\hline \multirow[t]{5}{*}{ IVC } & Urban & $0.043(-1.420)$ & $\begin{array}{l}0.0733^{* *} \\
(-2.450)\end{array}$ & $\begin{array}{l}0.0748^{* *} \\
(-2.500)\end{array}$ & $\begin{array}{l}0.0803^{* * *} \\
(-2.680)\end{array}$ & $\begin{array}{l}0.0695^{* *} \\
(-2.330)\end{array}$ & $\begin{array}{l}0.0695^{* *} \\
(-2.330)\end{array}$ & $0.054^{*}(-1.760)$ \\
\hline & Gender & $\begin{array}{l}0.245^{* * *} \\
(-6.670)\end{array}$ & $\begin{array}{l}0.231^{* * *} \\
(-6.300)\end{array}$ & $\begin{array}{l}0.236^{* * *} \\
(-6.430)\end{array}$ & $\begin{array}{l}0.230 * * * \\
(-6.270)\end{array}$ & $\begin{array}{l}0.238^{* * *} \\
(-6.480)\end{array}$ & $\begin{array}{l}0.235^{* * *} \\
(-6.400)\end{array}$ & $\begin{array}{l}0.230^{* * *} \\
(-6.250)\end{array}$ \\
\hline & Age & $\begin{array}{l}-0.0322^{* * *} \\
(-27.89)\end{array}$ & $\begin{array}{l}-0.0321^{* * *} \\
(-27.72)\end{array}$ & $\begin{array}{l}-0.0327^{* * *} \\
(-28.22)\end{array}$ & $\begin{array}{l}-0.0335^{* * *} \\
(-28.69)\end{array}$ & $\begin{array}{l}-0.0324^{* * *} \\
(-28.02)\end{array}$ & $\begin{array}{l}-0.0325^{* * *} \\
(-28.06)\end{array}$ & $\begin{array}{l}-0.0335^{* * *} \\
(-28.49)\end{array}$ \\
\hline & Marriage & $0.041(-1.050)$ & $0.051(-1.330)$ & $0.051(-1.330)$ & $0.050(-1.310)$ & $0.046(-1.200)$ & $0.051(-1.330)$ & $0.060(-1.550)$ \\
\hline & Education & $\begin{array}{l}0.0186^{* * *} \\
(-5.140)\end{array}$ & $\begin{array}{l}0.0243^{* * *} \\
(-6.920)\end{array}$ & $\begin{array}{l}0.0216^{* * *} \\
(-6.160)\end{array}$ & $\begin{array}{l}0.0254^{* * *} \\
(-7.180)\end{array}$ & $\begin{array}{l}0.0223^{* * *} \\
(-6.350)\end{array}$ & $\begin{array}{l}0.0226 * * * \\
(-6.450)\end{array}$ & $\begin{array}{l}0.0221^{* * *} \\
(-6.050)\end{array}$ \\
\hline \multirow[t]{4}{*}{ SCV } & Employ & $\begin{array}{l}0.238^{* * *} \\
(-6.830)\end{array}$ & $\begin{array}{l}0.238^{* * *} \\
(-6.800)\end{array}$ & $\begin{array}{l}0.235^{* * *} \\
(-6.700)\end{array}$ & $\begin{array}{l}0.237^{* * *} \\
(-6.780)\end{array}$ & $\begin{array}{l}0.239^{* * *} \\
(-6.820)\end{array}$ & $\begin{array}{l}0.241^{* * *} \\
(-6.880)\end{array}$ & $\begin{array}{l}0.237^{* * *} \\
(-6.770)\end{array}$ \\
\hline & Insurance & $\begin{array}{l}0.056^{* *} \\
(-0.130)\end{array}$ & $\begin{array}{l}0.012^{* *} \\
(-0.030)\end{array}$ & $\begin{array}{l}0.016^{*} \\
(-0.040)\end{array}$ & $\begin{array}{l}0.017^{* *} \\
(-0.040)\end{array}$ & $\begin{array}{l}0.013^{* *} \\
(-0.030)\end{array}$ & $\begin{array}{l}0.016^{*} \\
(-0.040)\end{array}$ & $0.093^{* *}(-0.230)$ \\
\hline & Family & $0.031(-0.640)$ & $0.039(-0.790)$ & $0.030(-0.610)$ & $0.033(-0.680)$ & $0.030(-0.620)$ & $0.034(-0.700)$ & $0.037(-0.760)$ \\
\hline & Status & $\begin{array}{l}0.0353^{* * *} \\
(-4.810)\end{array}$ & $\begin{array}{l}0.0257^{* * *} \\
(-3.560)\end{array}$ & $\begin{array}{l}0.0292 * * * \\
(-4.060)\end{array}$ & $\begin{array}{l}0.0311^{* * *} \\
(-4.320)\end{array}$ & $\begin{array}{l}0.0301 * * * \\
(-4.190)\end{array}$ & $\begin{array}{l}0.0316^{* * *} \\
(-4.360)\end{array}$ & $\begin{array}{l}0.034^{* * *} \\
(-4.610)\end{array}$ \\
\hline \multirow[t]{4}{*}{ Living habit } & Exercise & $\begin{array}{l}0.017^{* * *} \\
(-1.390)\end{array}$ & $\begin{array}{l}0.016^{* *} \\
(-1.280)\end{array}$ & $\begin{array}{l}0.014^{* *} \\
(-1.120)\end{array}$ & $\begin{array}{l}0.015^{* * *} \\
(-1.160)\end{array}$ & $\begin{array}{l}0.012^{* *} \\
(-0.970)\end{array}$ & $\begin{array}{l}0.016^{* *} \\
(-1.310)\end{array}$ & $0.010^{*}(-0.750)$ \\
\hline & Smoke & $\begin{array}{l}-0.010^{* *} \\
(-2.48)\end{array}$ & $\begin{array}{l}-0.008^{*} \\
(-1.96)\end{array}$ & $\begin{array}{l}-0.010^{* *} \\
(-2.37)\end{array}$ & $\begin{array}{l}-0.009^{* *} \\
(-2.22)\end{array}$ & $\begin{array}{l}-0.010^{* *} \\
(-2.32)\end{array}$ & $\begin{array}{l}-0.009^{* *} \\
(-2.26)\end{array}$ & $\begin{array}{l}-0.009^{* *} \\
(-2.16)\end{array}$ \\
\hline & Drink & $\begin{array}{l}0.109^{* * *} \\
(-2.790)\end{array}$ & $\begin{array}{l}0.109^{* * *} \\
(-2.780)\end{array}$ & $\begin{array}{l}0.113^{* * *} \\
(-2.890)\end{array}$ & $\begin{array}{l}0.115^{* * *} \\
(-2.940)\end{array}$ & $\begin{array}{l}0.109^{* * *} \\
(-2.810)\end{array}$ & $\begin{array}{l}0.111^{* * *} \\
(-2.830)\end{array}$ & $\begin{array}{l}0.117^{* * *} \\
(-3.000)\end{array}$ \\
\hline & Break & $\begin{array}{l}0.291^{* * *} \\
(-6.910)\end{array}$ & $\begin{array}{l}0.299 * * * \\
(-7.100)\end{array}$ & $\begin{array}{l}0.296 * * * \\
(-7.040)\end{array}$ & $\begin{array}{l}0.293^{* * *} \\
(-6.970)\end{array}$ & $\begin{array}{l}0.294^{* * *} \\
(-6.990)\end{array}$ & $\begin{array}{l}0.296^{* * *} \\
(-7.020)\end{array}$ & $\begin{array}{l}0.299 * * * \\
(-7.060)\end{array}$ \\
\hline \multirow[t]{2}{*}{ ME } & Satisfaction & $\begin{array}{l}0.102^{* * *} \\
(-3.62)\end{array}$ & $\begin{array}{l}0.0966^{* * *} \\
(-3.42)\end{array}$ & $\begin{array}{l}0.0998^{* * *} \\
(-3.53)\end{array}$ & $\begin{array}{l}0.0982^{* * *} \\
(-3.48)\end{array}$ & $\begin{array}{l}0.0996 * * * \\
(-3.53)\end{array}$ & $\begin{array}{l}0.0987^{* * *} \\
(-3.49)\end{array}$ & $0.097^{* * *}(-3.43)$ \\
\hline & Level & $\begin{array}{l}0.0578^{* * *} \\
(-2.800)\end{array}$ & $\begin{array}{l}0.0556^{* * *} \\
(-2.700)\end{array}$ & $\begin{array}{l}0.0477^{* *} \\
(-2.300)\end{array}$ & $\begin{array}{l}0.0480^{* *} \\
(-2.330)\end{array}$ & $\begin{array}{l}0.0567^{* * *} \\
(-2.750)\end{array}$ & $\begin{array}{l}0.0561^{* * *} \\
(-2.720)\end{array}$ & $0.039 *(-1.870)$ \\
\hline Observations & & 22,751 & 22,751 & 22,751 & 22,751 & 22,751 & 22,751 & 22,751 \\
\hline Province & & YES & YES & YES & YES & YES & YES & YES \\
\hline Prob $>\mathrm{F}$ & & 0.000 & 0.000 & 0.000 & 0.000 & 0.000 & 0.000 & 0.000 \\
\hline Pseudo $R^{2}$ & & 0.055 & 0.055 & 0.055 & 0.055 & 0.054 & 0.054 & 0.057 \\
\hline
\end{tabular}


significantly negative $(\beta=-0.964, \quad \mathrm{OR}=0.382$ and $\beta=-0.381$, OR $=0.758 ; P<0.01)$. These results show that IIequality has a negative effect on $\mathrm{PH}$ and $\mathrm{MH}$.

\section{The impact of social capital on physical and mental health}

This study revealed that cognitive $\mathrm{SC}$ has a positive effect on $\mathrm{PH}$ and $\mathrm{MH}$. As shown in columns (2)-(6) of Tables 2 and 3, the coefficients of cognitive SC on $\mathrm{PH}$ are 0.0471 and $0.0674(\mathrm{OR}=1.048 ; \mathrm{OR}=1.070$, $P<0.01$ ), and the coefficients on $\mathrm{MH}$ are 0.150 and $0.0977(\mathrm{OR}=1.162$; OR $=1.103, P<0.01)$. Structural SC also shows a significantly effect on $\mathrm{PH}$ and $\mathrm{MH}$, with the coefficients on $\mathrm{MH}$ being $0.002(\mathrm{OR}=1.002, P>0.1)$, $0.028(\mathrm{OR}=1.028, P<0.01)$, and $0.029(\mathrm{OR}=1.030$, $P<0.01)$, respectively, and the regression coefficients on $\mathrm{PH}$ being $-0.024 \quad(\mathrm{OR}=0.976, \quad P<0.01), 0.009$ $(\mathrm{OR}=1.009, \quad P>0.1), \quad-0.010 \quad(\mathrm{OR}=0.990, \quad P<0.01)$. Integrating other variables (column (7)), the significance level of the regression coefficient is still high (Cognitive $\mathrm{SC}(\mathrm{MH}): \beta=0.146$ and $0.104, \mathrm{OR}=1.157$ and 1.110, $P<0.01$; Cognitive SC $(\mathrm{PH}): \beta=0.0460$ and $0.0687, \mathrm{OR}=1.047$ and 1.071, $P<0.01$; Structural $\mathrm{SC}$ $(\mathrm{MH}): \beta=-0.005,0.025$ and $0.015, \mathrm{OR}=0.995,1.025$ and 1.015, $P>0.1, P<0.01$ and $P<0.01$; Structural SC $(\mathrm{PH}): \beta=-0.026,0.009$ and $-0.013, \mathrm{OR}=0.975,1.009$ and 0.987, $P<0.01, P>0.1$ and $P<0.01$ ). The regression results can fully support that cognitive SC and structural SC can have effects on promoting the development of $\mathrm{PH}$ and $\mathrm{MH}$.

\section{The impact of covariates on physical and mental health}

The columns (7) of Tables 2 and 3 show that household registration and gender have a significantly positive correlation with $\mathrm{PH}$ and $\mathrm{MH}$ (COA: 0.054, 0.230, $\mathrm{OR}=1.056$ and $1.258 ; 0.113,0.480, \mathrm{OR}=1.120$ and 1.616; $P<0.01 ; \mathrm{COA}=$ Coefficient of action). These results indicate that there are household registration differences and gender differences in the inhibitory effect of IIequality on health. Factors such as the level of medical insurance and social status play a positive role in regulating $\mathrm{PH}$ and $\mathrm{MH}$ (COA: 0.093, 0.034, $\mathrm{OR}=1.097$ and $1.035 ; 0.112,0.048, \mathrm{OR}=1.119$ and 1.049; $P<0.01)$. Good living habits also have a positive effect on $\mathrm{PH}$ and $\mathrm{MH}$, including not smoking, regular exercise, and lunch breaks. People should be encouraged to develop good habits. The higher the level of medical service satisfaction and diagnosis and treatment, the more beneficial to $\mathrm{PH}$ and $\mathrm{MH}$ (COA: 0.097, $0.039, \mathrm{OR}=0.907$ and $1.040 ; 0.076,0.116$, OR $=1.079$ and 1.123; $P<0.01$ ), indicating the importance of the improvement of the medical environment and the level of diagnosis and treatment.

\section{Robustness test}

Above all, the goodness of fit of the ordered logistic regression equation was analyzed. The values of Pseudo $R^{2}$ are maintained between $0.04-0.06$, and the probability calculated by the Log-likelihood test is 0 , which is less than the significance level of 0.05 (Prob $>$ F). It shows that the selected IV has a significant influence on the DV, and the overall fit of the equation is good.

The results from various robustness tests are summarized in Table 4. Taking the change of the DV as an example, the effective coefficient of IIequality on self-rated health is $-0.558(\mathrm{OR}=0.572)$, and the effect coefficient is negative. The coefficients of social trust, social justice, social support, interpersonal relationship, network density and other SC values are 0.080, 0.037, - 0.005, 0.032, -0.004 , respectively, which are proved to be significant $(\mathrm{OR}=1.083,1.037,0.995,1.032,0.996)$. According to the results of a variety of robustness tests, Ilequality will inhibit $\mathrm{PH}$ and $\mathrm{MH}$, and $\mathrm{SC}$ plays an important role in $\mathrm{PH}$ and $\mathrm{MH}$. This conclusion is stable and reliable.

\section{Heterogeneity validation}

The first four columns of Table 5 show that Ilequality has a negative effect on the $\mathrm{PH}$ and $\mathrm{MH}$ of both men and women but is more prominent in the female group. In most circumstances, $\mathrm{SC}$ has a positive effect on the $\mathrm{PH}$ and $\mathrm{MH}$ of both sexes, but its boosting effect on health performs better in the male group. As shown in the last four columns of Table 5, the deprivation effect of IIequality on the $\mathrm{PH}$ and $\mathrm{MH}$ of rural residents is higher than that of urban residents, but the overall gap is not significantly different. Different SC showed slightly different performance in different household registration groups. Cognitive SC had a greater positive effect on the $\mathrm{PH}$ and $\mathrm{MH}$ of rural residents, while structural SC exhibited a more obvious effect on the $\mathrm{PH}$ and $\mathrm{MH}$ of urban residents. Table 6 shows the analysis results showing the differences by region. According to China's three major economic divisions, the region is divided into eastern, central, and western regions, and then the regional heterogeneity was regressed and calculated. The regression results show that the inhibitory effect of Ilequality on the $\mathrm{PH}$ and $\mathrm{MH}$ of the population in different regions showed a decreasing trend from east to middle and west. There are also some regional differences in the effects of $\mathrm{SC}$ on $\mathrm{PH}$ and $\mathrm{MH}$, but the effect was more significant on the population in the eastern region.

\section{Intermediary effect validation}

The mediation effect in this study was designed to analyze the data $(N=22,751)$ by the KHB method. As shown in Table 7, it can be observed that there is a partial 
Table 4 Summary results of robustness analysis

\begin{tabular}{|c|c|c|c|c|c|c|c|c|}
\hline \multirow[t]{3}{*}{ Variable } & & (1) & $(2)$ & (3) & (4) & $(5)$ & (6) & (7) \\
\hline & & $\begin{array}{l}\text { Health } \\
\text { variable }\end{array}$ & MH & $\mathrm{PH}$ & MH & $\mathrm{PH}$ & MH & $\mathrm{PH}$ \\
\hline & & $\begin{array}{l}\text { Change DV } \\
\text { (Self-rated) }\end{array}$ & \multicolumn{2}{|c|}{ Change IV (Podder index) } & \multicolumn{2}{|c|}{ Outlier test (Winsor method) } & \multicolumn{2}{|c|}{$\begin{array}{l}\text { Change estimation model } \\
\text { (OLS) }\end{array}$} \\
\hline Ilequality & & $\begin{array}{l}-0.558^{* * *} \\
(-8.75)\end{array}$ & $\begin{array}{l}-2.052^{* * *} \\
(-9.390)\end{array}$ & $\begin{array}{l}-6.535^{* * *} \\
(-21.500)\end{array}$ & $\begin{array}{l}-0.970^{* * *} \\
(-14.24)\end{array}$ & $\begin{array}{l}-0.378^{* * *} \\
(-5.38)\end{array}$ & $\begin{array}{l}-2.104^{* * *} \\
(-15.47)\end{array}$ & $\begin{array}{l}-0.147^{* * *} \\
(-5.34)\end{array}$ \\
\hline \multirow[t]{5}{*}{ SC } & Trust & $\begin{array}{l}0.080^{* * *} \\
(-7.490)\end{array}$ & $\begin{array}{l}0.147^{* * *} \\
(-13.080)\end{array}$ & $\begin{array}{l}0.042^{* * *} \\
(-3.660)\end{array}$ & $\begin{array}{l}0.148^{* * *} \\
(-13.160)\end{array}$ & $\begin{array}{l}0.048^{* * *} \\
(-4.180)\end{array}$ & $\begin{array}{l}0.304^{* * *} \\
(-13.570)\end{array}$ & $\begin{array}{l}0.017^{* * *} \\
(-3.770)\end{array}$ \\
\hline & Fair & $\begin{array}{l}0.037^{* * *} \\
(-4.090)\end{array}$ & $\begin{array}{l}0.100^{* * *} \\
(-10.900)\end{array}$ & $\begin{array}{l}0.066^{* * *} \\
(-6.860)\end{array}$ & $\begin{array}{l}0.106^{* * *} \\
(-11.580)\end{array}$ & $\begin{array}{l}0.070^{* * *} \\
(-7.370)\end{array}$ & $\begin{array}{l}0.211^{* * *} \\
(-11.820)\end{array}$ & $\begin{array}{l}0.024^{* * *} \\
(-6.580)\end{array}$ \\
\hline & Support & $\begin{array}{l}-0.005^{*} \\
(-1.66)\end{array}$ & $0.004(-1.270)$ & $\begin{array}{l}-0.014^{* * *} \\
(-3.93)\end{array}$ & $-0.005(-1.43)$ & $\begin{array}{l}-0.026^{* * *} \\
(-7.26)\end{array}$ & $\begin{array}{l}-0.017^{* * *} \\
(-2.59)\end{array}$ & $\begin{array}{l}-0.010^{* * *} \\
(-7.28)\end{array}$ \\
\hline & Relations & $\begin{array}{l}0.032^{* * *} \\
(-4.760)\end{array}$ & $\begin{array}{l}0.026^{* * *} \\
(-3.730)\end{array}$ & $\begin{array}{l}0.013^{* *} \\
(-1.730)\end{array}$ & $\begin{array}{l}0.025^{* * *} \\
(-3.640)\end{array}$ & $0.010(-1.350)$ & $\begin{array}{l}0.055^{* * *} \\
(-4.030)\end{array}$ & $0.004^{* *}(-1.340)$ \\
\hline & Jiegousc & $\begin{array}{l}-0.004^{*} \\
(-0.71)\end{array}$ & $\begin{array}{l}0.034^{* * *} \\
(-6.040)\end{array}$ & $\begin{array}{l}-0.010^{* *} \\
(-1.750)\end{array}$ & $\begin{array}{l}0.0138^{* *} \\
(-2.410)\end{array}$ & $\begin{array}{l}-0.0133^{* *} \\
(-2.22)\end{array}$ & $\begin{array}{l}0.0281^{* *} \\
(-2.420)\end{array}$ & $-0.004(-1.57)$ \\
\hline CV & & Yes & Yes & Yes & Yes & Yes & Yes & Yes \\
\hline PDV & & Yes & Yes & Yes & Yes & Yes & Yes & Yes \\
\hline Observations & & 22,751 & 22,750 & 22,750 & 22,296 & 22,296 & 22,751 & 22,751 \\
\hline Prob $>\mathrm{F}$ & & 0.000 & 0.000 & 0.000 & 0.000 & 0.000 & 0.000 & 0.000 \\
\hline Pseudo $\mathrm{R}^{2}$ & & 0.051 & 0.114 & 0.121 & 0.048 & 0.058 & 0.046 & 0.073 \\
\hline
\end{tabular}

MH Mental health, PH Physical health, DV Dependent variable, IV Independent Variable, OLS Ordinary Least Squares Regression, Ilequality Income inequality, SC Social capital, CV Control variable, PDV Province dummy variablet

${ }^{* *} P<0.01$, ${ }^{*} P<0.05,{ }^{*} P<0.1$, robust $t$ statistic reported in brackets

mediating effect of social capital collection in the path of IIequality on $\mathrm{PH}$ and $\mathrm{MH}$. After controlling for confounding variables such as CVs and PDV, the mediating effect of cognitive SC in the total effect of income inequality on physical and mental health accounted for $6.95,4.59,3.44,2.74 \%(\mathrm{OR}=0.919,0.055,0.977,0.981$;

Table 5 Test results of gender and household registration heterogeneity

\begin{tabular}{|c|c|c|c|c|c|c|c|c|c|}
\hline \multirow[t]{3}{*}{ Variable } & & (1) & (2) & (3) & (4) & (5) & (6) & (7) & (8) \\
\hline & & $\mathrm{MH}$ & $\mathrm{PH}$ & $\mathrm{MH}$ & $\mathrm{PH}$ & $\mathrm{MH}$ & $\mathrm{PH}$ & $\mathrm{MH}$ & $\mathrm{PH}$ \\
\hline & & \multicolumn{2}{|l|}{ Female } & \multicolumn{2}{|l|}{ Male } & \multicolumn{2}{|l|}{ Rural } & \multicolumn{2}{|l|}{ Urban } \\
\hline Ilequality & & $\begin{array}{l}-1.047^{* * *} \\
(-11.13)\end{array}$ & $\begin{array}{l}-0.387^{* * *} \\
(-4.06)\end{array}$ & $\begin{array}{l}-0.915^{* * *} \\
(-9.27)\end{array}$ & $\begin{array}{l}-0.386^{* * *} \\
(-3.81)\end{array}$ & $\begin{array}{l}-0.957^{* * *} \\
(-10.16)\end{array}$ & $\begin{array}{l}-0.319^{* * *} \\
(-3.23)\end{array}$ & $\begin{array}{l}-0.930^{* * *} \\
(-9.27)\end{array}$ & $\begin{array}{l}-0.273^{* * *} \\
(-4.59)\end{array}$ \\
\hline \multirow[t]{5}{*}{ SC } & Trust & $\begin{array}{l}0.134^{* * *} \\
(-8.650)\end{array}$ & $\begin{array}{l}0.0269^{*} \\
(-1.720)\end{array}$ & $\begin{array}{l}0.158^{* * *} \\
(-9.790)\end{array}$ & $\begin{array}{l}0.0637^{* * *} \\
(-3.880)\end{array}$ & $\begin{array}{l}0.162^{* * *} \\
(-9.320)\end{array}$ & $\begin{array}{l}0.0493^{* * *} \\
(-2.800)\end{array}$ & $\begin{array}{l}0.153^{* * *} \\
(-9.450)\end{array}$ & $\begin{array}{l}0.0488^{* * *} \\
(-2.940)\end{array}$ \\
\hline & Fair & $\begin{array}{l}0.102^{* * *} \\
(-8.180)\end{array}$ & $\begin{array}{l}0.0643^{* * *} \\
(-5.040)\end{array}$ & $\begin{array}{l}0.106^{* * *} \\
(-8.020)\end{array}$ & $\begin{array}{l}0.0713^{* * *} \\
(-5.120)\end{array}$ & $\begin{array}{l}0.115^{* * *} \\
(-9.320)\end{array}$ & $\begin{array}{l}0.0902^{* * *} \\
(-3.950)\end{array}$ & $\begin{array}{l}0.0886^{* * *} \\
(-6.610)\end{array}$ & $\begin{array}{l}0.0899^{* * *} \\
(-6.390)\end{array}$ \\
\hline & Support & $\begin{array}{l}0.000 \\
(-0.100)\end{array}$ & $\begin{array}{l}-0.030^{* * *} \\
(-6.03)\end{array}$ & $\begin{array}{l}-0.0101^{* *} \\
(-2.06)\end{array}$ & $\begin{array}{l}-0.0209^{* * *} \\
(-3.98)\end{array}$ & $\begin{array}{l}-0.003 \\
(-0.68)\end{array}$ & $\begin{array}{l}-0.0125^{* *} \\
(-2.49)\end{array}$ & $\begin{array}{l}-0.004 \\
(-0.80)\end{array}$ & $\begin{array}{l}-0.037^{* * *} \\
(-7.38)\end{array}$ \\
\hline & Relations & $\begin{array}{l}0.011 \\
(-1.180)\end{array}$ & $\begin{array}{l}0.000 \\
(-0.010)\end{array}$ & $\begin{array}{l}0.040^{* * *} \\
(-4.040)\end{array}$ & $\begin{array}{l}0.022^{* *} \\
(-2.130)\end{array}$ & $\begin{array}{l}0.022^{* *} \\
(-2.370)\end{array}$ & $\begin{array}{l}0.002 \\
(-0.170)\end{array}$ & $\begin{array}{l}0.028^{* * *} \\
(-2.800)\end{array}$ & $\begin{array}{l}0.018^{*} \\
(-1.740)\end{array}$ \\
\hline & Jiegousc & $\begin{array}{l}0.013 \\
(-1.630)\end{array}$ & $\begin{array}{l}-0.020^{* *} \\
(-2.40)\end{array}$ & $\begin{array}{l}0.017^{* *} \\
(-2.040)\end{array}$ & $\begin{array}{l}-0.007 \\
(-0.81)\end{array}$ & $\begin{array}{l}0.013^{* *} \\
(-1.510)\end{array}$ & $\begin{array}{l}-0.023^{* *} \\
(-2.53)\end{array}$ & $\begin{array}{l}0.014^{*} \\
(-1.650)\end{array}$ & $\begin{array}{l}-0.028^{* *} \\
(-1.18)\end{array}$ \\
\hline CV & & Yes & Yes & Yes & Yes & Yes & Yes & Yes & Yes \\
\hline PDV & & Yes & Yes & Yes & Yes & Yes & Yes & Yes & Yes \\
\hline Observations & & 11,879 & 11,879 & 10,872 & 10,872 & 11,546 & 11,546 & 11,205 & 11,205 \\
\hline Prob $>\mathrm{F}$ & & 0.000 & 0.000 & 0.000 & 0.000 & 0.000 & 0.000 & 0.000 & 0.000 \\
\hline Pseudo $\mathrm{R}^{2}$ & & 0.042 & 0.058 & 0.046 & 0.051 & 0.049 & 0.062 & 0.042 & 0.056 \\
\hline
\end{tabular}

MH Mental health, $P H$ Physical health, llequality Income inequality, SC Social capital, CV Control variable, PDV Province dummy variablet **** $P<0.01$, ** $P<0.05, * P<0.1$, robust t statistic reported in brackets 
Table 6 Results of regional heterogeneity test

\begin{tabular}{|c|c|c|c|c|c|c|c|}
\hline \multirow[t]{3}{*}{ Variable } & & (1) & $(2)$ & (3) & (4) & (5) & (6) \\
\hline & & $\mathrm{MH}$ & $\mathrm{PH}$ & MH & $\mathrm{PH}$ & MH & $\mathrm{PH}$ \\
\hline & & \multicolumn{2}{|l|}{ Eastern region } & \multicolumn{2}{|l|}{ Central region } & \multicolumn{2}{|l|}{ Western region } \\
\hline Ilequality & & $-1.104^{* * *}(-11.27)$ & $-0.529^{* * *}(-5.24)$ & $-1.088^{* * *}(-7.12)$ & $-0.352^{* *}(-2.24)$ & $-0.693^{* * *}(-5.71)$ & $-0.207^{*}(-1.64)$ \\
\hline \multirow[t]{5}{*}{ SC } & Trust & $0.148^{* * *}(-9.280)$ & $0.047^{* * *}(-2.900)$ & $0.123^{* * *}(-4.870)$ & $0.038(-1.460)$ & $0.147^{* * *}(-7.410)$ & $0.0359^{*}(-1.780)$ \\
\hline & Fair & $0.125^{* * *}(-7.950)$ & $0.093^{* * *}(-6.730)$ & $0.115^{* * *}(-5.740)$ & $0.055^{* * *}(-2.680)$ & $0.097^{* * *}(-6.000)$ & $0.042^{* *}(-2.550)$ \\
\hline & Support & $-0.011^{* *}(-2.23)$ & $-0.028^{* * *}(-5.51)$ & $-0.002(-0.22)$ & $-0.030^{* * *}(-3.88)$ & $0.005(-0.760)$ & $-0.017^{* * *}(-2.63)$ \\
\hline & Relations & $0.048^{* * *}(-4.750)$ & $0.001(-0.130)$ & $0.024^{*}(-1.640)$ & $0.014(-0.850)$ & $-0.007(-0.60)$ & $0.017(-1.440$ \\
\hline & Jiegousc & $0.0123^{*}(-1.650)$ & $-0.008^{*}(-1.08)$ & $0.002^{*}(-0.130)$ & $-0.036^{* *}(-2.45)$ & $0.026^{* *}(-2.180)$ & $-0.015(-1.26)$ \\
\hline CV & & Yes & Yes & Yes & Yes & Yes & Yes \\
\hline PDV & & Yes & Yes & Yes & Yes & Yes & Yes \\
\hline Observations & & 10,952 & 10,952 & 4,926 & 4,926 & 6,873 & 6,873 \\
\hline Prob $>\mathrm{F}$ & & 0.000 & 0.000 & 0.000 & 0.000 & 0.000 & 0.000 \\
\hline Pseudo $R^{2}$ & & 0.046 & 0.054 & 0.037 & 0.059 & 0.043 & 0.060 \\
\hline
\end{tabular}

MH Mental health, $P H$ Physical health, Ilequality Income inequality, SC Social capital, $C V$ Control variable, $P D V$ Province dummy variablet

${ }^{* * *} P<0.01,{ }^{* *} P<0.05,{ }^{*} P<0.1$, robust t statistic reported in brackets

$P<0.001)$. As for the three structural SCs, the IE of Network density on the association of Ilequality with $\mathrm{MH}$ was $2.98 \%(\mathrm{OR}=0.965, P<0.001)$, at the same time, the IE of Social support on the association of Ilequality with $\mathrm{PH}$ was $13.22 \%(\mathrm{OR}=1.095, P<0.001)$.

\section{Discussion}

Although it is not uncommon to apply the KI to the measurement of Ilequality [40, 41], this study extended it to the collaborative study of IIequality, SC, and PH and $\mathrm{MH}$ for the first time. Especially, this paper has applied this index to data from a developing country milieu through more sophisticated regression models.

The outcome of the research provides important support to demonstrate the negative impact of Ilequality on $\mathrm{PH}$ and $\mathrm{MH}$. After controlling the individual characteristics, family characteristics, medical service quality, and $\mathrm{SC}$ variables that affect $\mathrm{PH}$ and $\mathrm{MH}$, we note that Ilequality will directly affect $\mathrm{PH}$ and $\mathrm{MH}$. However, the results reveal that the negative effect of Ilequality on the mental health of an individual is more obvious than the negative effects on physical health. Consistent with the extant works, the conclusions of this study support the hypothesis that as IIequality increases, the individual's physical and mental health will decline. From the theoretical point of view, Ilequality will deprive low-income people of their ability to obtain medical resources which leads to a diminution of the quality of the living environment which can directly affect their health [42]. At the same time, Ilequality stimulates a serious social and psychological shadow, which can cause anxiety and stress and ultimately damage $\mathrm{MH}$ [10]. The empirical results are consistent with the relative income hypothesis and income deprivation hypothesis. With the development of complex social situations such as economic globalization, miniaturization of family populations, and aging population aging, more attention needs to be paid to the adverse consequences caused by IIequality [43] and improvement measures. This study has demonstrated the positive effect of $\mathrm{SC}$ on $\mathrm{PH}$ and $\mathrm{MH}$ and shown that the coefficients of the five $\mathrm{SC}$ indicators are positive in most circumstances, suggesting the correlation of SC with the $\mathrm{PH}$ and $\mathrm{MH}$. A novelty in this study is that when $\mathrm{SC}$ was decomposed into two identifiable forms, the study affirmed the fact that shows that both cognitive SC and structural SC can influence $\mathrm{PH}$ and $\mathrm{MH}$, which is consistent with the research conclusions of other scholars [44]. As an informal system, SC can guide more social participation and social activities, and promote the development of $\mathrm{MH}$ and healthy behaviors $[45,46]$.

IIequality at the individual level can reflect the relative degree of deprivation of income. From the comparison of the coefficient values of the individual IIequality effect and the Ilequality effect of other variables of cointegration, it is revealed that the negative effect of the cointegration coefficient is more obvious $(-0.964<-0.962$; $-0.381<-0.280 ; \quad$ OR $=0.382, \quad 0.382, \quad 0.758, \quad 0.756$; $P<0.01)$. The results of this study strengthen the explanatory effect of relative deprivation on the theory of IIequality. Relative deprivation can affect $\mathrm{PH}$ and $\mathrm{MH}$ through material pathways, psychological pathways, and acting on SC [5]. The path of Ilequality on $\mathrm{PH}$ and 
Table 7 Test results of the mediation effect of social capital

\begin{tabular}{|c|c|c|c|c|c|c|c|c|}
\hline DV & IV & & Coef. & OR & Std. Err. & $z$ & $P>|z|$ & $\begin{array}{l}\text { Mediation } \\
\text { percentage }\end{array}$ \\
\hline \multirow[t]{15}{*}{ MH } & Sense of Social trust & TE & -1.211 & 0.298 & 0.052 & -23.330 & 0.000 & $6.95 \%$ \\
\hline & & $\mathrm{DE}$ & -1.127 & 0.324 & 0.052 & -21.670 & 0.000 & \\
\hline & & $\mathrm{IE}$ & -0.084 & 0.919 & 0.008 & -10.230 & 0.000 & \\
\hline & Sense of Social fairness & TE & -1.205 & 0.300 & 0.052 & -23.300 & 0.000 & $4.59 \%$ \\
\hline & & $\mathrm{DE}$ & -1.260 & 0.284 & 0.052 & -24.170 & 0.000 & \\
\hline & & $\mathrm{IE}$ & 0.055 & 1.057 & 0.007 & 8.220 & 0.000 & \\
\hline & Social support & TE & -1.201 & 0.301 & 0.052 & -23.240 & 0.000 & $1.21 \%$ \\
\hline & & $\mathrm{DE}$ & -1.216 & 0.297 & 0.053 & -23.150 & 0.000 & \\
\hline & & $\mathrm{IE}$ & 0.015 & 1.015 & 0.009 & 1.570 & 0.117 & \\
\hline & Personal relationship & $\mathrm{TE}$ & -1.202 & 0.301 & 0.052 & -23.240 & 0.000 & $0.10 \%$ \\
\hline & & $\mathrm{DE}$ & -1.200 & 0.301 & 0.052 & -23.220 & 0.000 & \\
\hline & & $\mathrm{IE}$ & -0.001 & 0.999 & 0.002 & -0.630 & 0.526 & \\
\hline & Network density & TE & -1.202 & 0.301 & 0.052 & -23.260 & 0.000 & $2.98 \%$ \\
\hline & & $\mathrm{DE}$ & -1.166 & 0.312 & 0.052 & -22.280 & 0.000 & \\
\hline & & $\mathrm{IE}$ & -0.036 & 0.965 & 0.009 & -4.080 & 0.000 & \\
\hline \multirow[t]{15}{*}{$\mathrm{PH}$} & Sense of Social trust & $\mathrm{TE}$ & -0.688 & 0.503 & 0.053 & -12.990 & 0.000 & $3.44 \%$ \\
\hline & & $\mathrm{DE}$ & -0.664 & 0.515 & 0.053 & -12.510 & 0.000 & \\
\hline & & $\mathrm{IE}$ & -0.024 & 0.977 & 0.005 & -5.190 & 0.000 & \\
\hline & Sense of Social fairness & TE & -0.687 & 0.503 & 0.053 & -12.980 & 0.000 & $2.74 \%$ \\
\hline & & $\mathrm{DE}$ & -0.668 & 0.513 & 0.053 & -12.550 & 0.000 & \\
\hline & & $\mathrm{IE}$ & -0.019 & 0.981 & 0.006 & -3.060 & 0.002 & \\
\hline & Social support & TE & -0.687 & 0.503 & 0.053 & -12.990 & 0.000 & $13.22 \%$ \\
\hline & & $\mathrm{DE}$ & -0.778 & 0.459 & 0.053 & -12.510 & 0.000 & \\
\hline & & $\mathrm{IE}$ & 0.091 & 1.095 & 0.005 & -5.190 & 0.000 & \\
\hline & Personal relationship & TE & -0.687 & 0.503 & 0.053 & -12.980 & 0.000 & $0.01 \%$ \\
\hline & & $\mathrm{DE}$ & -0.687 & 0.503 & 0.053 & -12.980 & 0.000 & \\
\hline & & $\mathrm{IE}$ & 0.000 & 1.000 & 0.000 & -0.360 & 0.720 & \\
\hline & Network density & TE & -0.687 & 0.503 & 0.053 & -12.980 & 0.000 & $1.00 \%$ \\
\hline & & $\mathrm{DE}$ & -0.680 & 0.507 & 0.054 & -12.680 & 0.000 & \\
\hline & & $\mathrm{IE}$ & -0.007 & 0.993 & 0.009 & -0.770 & 0.443 & \\
\hline
\end{tabular}

Mediation percentage is calculated by IE/TE $\times 100$

DV Dependent variable, IV Independent Variable, $M H$ Mental health, $P H$ Physical health, TE Total effect, DE Direct effect, IE Intermediary effect

$\mathrm{MH}$ may involve the interaction of multiple intermediary variables, which may result in differences in the COA due to intermediary confusion and ranking issues [47]. A coherent study of Ilequality, $\mathrm{SC}$ and $\mathrm{PH}$ and $\mathrm{MH}$ is needed to further clarify the mediating role of SC in the path of Ilequality on $\mathrm{PH}$ and $\mathrm{MH}$. Our results show that both cognitive SC (social justice and social trust) and structural SC (social support and network density) have IEs. It is necessary to guard against the erosion of SC caused by Ilequality, which may result in a double cumulative effect of income disadvantage and social resource disadvantage [48].

It is interesting to note that women's $\mathrm{PH}$ and $\mathrm{MH}$ are more vulnerable to the negative effects of IIequality relative to that of the men, but the positive effects of SC are not obvious. The cause of this observation may be due to differences in physiology and social status. In terms of education, work, social resources and other aspects, women' opportunities are more unequal than men's, resulting in women's income disadvantage and lack of social resources [49]. The same problem also appears between rural and urban residents. The deprivation effect of Ilequality on the $\mathrm{PH}$ and $\mathrm{MH}$ of rural residents is higher than that of urban residents, but the effect of rural residents' structural SC on health promotion is still at a disadvantage. This may be due to China's unique urban-rural dual structure. Because of the nature of the institutional division of urban and rural areas, there are differences in wealth accumulation and income channels among urban 
and rural residents [50]. And the institutional dividend, family and social resources of urban residents are still significantly better than those in rural areas [51]. More attention needs to be paid to the heterogeneity of the impact of different influencing factors on $\mathrm{PH}$ and $\mathrm{MH}$ and take targeted measures for different groups of people. This is where follow-up research needs to be deepened.

This study used China's 2018 CFPS, a typical representative micro-database for empirical analysis. The data sample size is large $(N=22,751)$ and the survey content focuses on many aspects such as society, income, people's livelihood, education, health, etc., all of which are firsthand data, ensuring the applicability of the research data. After multiple rounds of problem correction, these data can truly reflect the $\mathrm{PH}$ and $\mathrm{MH}$ of Chinese people and related influencing factors, laying a foundation for the reliability of research conclusions. The research comprehensively adopts the robustness test method to repeatedly validate the research conclusions, including methods such as replacing IV or DV, changing the estimation model, and winsor's tailing treatment method. The research conclusions are consistent, indicating the stability and reliability of the research conclusions. Finally, The KHB analysis results prove that after controlling for variables such as individual characteristics, social characteristics, and living habits, SC does have a partial mediating role in the path of Ilequality on $\mathrm{PH}$ and $\mathrm{MH}$ [44]. In addition, the study did not only analyze the impact of overall sample data on $\mathrm{PH}$ and $\mathrm{MH}$, but also conducted partial analysis by gender, household registration, and population, and discusses the differences in factors affecting $\mathrm{PH}$ and $\mathrm{MH}$ of different populations, which is an expansion from the whole to the part.

Research findings might provide some enlightenment for developing countries to improve national health. At first, we should attach great importance to the weakening effect of inequality on physical and mental health, pay attention to income equity, be alert to the vicious circle of health-poverty traps, and take active interventions to decouple income from health [2, 3]; Secondly, take targeted measures for the physical and mental health of groups with special difficulties. Female population [49], rural population and poor population in underdeveloped areas are the focus of attention [50,51]. For this group of people, there is a need to strengthen the construction of a preferential social security system, increase educational opportunities, and pay attention to employment fairness; Finally, it is important to actively play an intermediary role of social capital, inequality buffer negative effects of physical and mental health. The development of SC, especially cognitive SC, can enhance people's perception of social fairness and trust, reduce resistance to economic development, enhance transaction transparency, provide active social supervision and management, and improve the efficiency of economic operations. Individuals should also attach importance to the construction and development of their social networks. Social networks can not only broaden the source of resource information, but also have the function of insurance apportionment, enhance the individual's ability to resist risks, provide individuals with emotional support and material support, and rely on social support and resources. Mutual assistance gets rid of the disadvantages of its development [18-20].

\section{Limitation}

This research also has a few limitations. Firstly, the data in this study are cross-sectional data. The conclusion can only explain the correlation between variables, and the direction of the influence path cannot be determined. There may be problems such as missing variables. At the same time, the reverse causality problem cannot be ruled out [52]. The impact of Ilequality on SC and PH and $\mathrm{MH}$ may have a time lag [53]. Studies have shown that there is a considerable lag time for the impact of Ilequality on health, and current Ilequality may have an important impact on later health [54]. This study did not include the influence of time frame on the research conclusions, which may limit the interpretation of the regression results. At the same time, the selected analysis indicators have a certain degree of subjectivity. For example, SC variables such as social justice and social trust are mostly subjective variables, and there are potential psychological characteristics to confuse the relationship between independent variables and dependent variables [55].

\section{Conclusions}

This study shows that Ilequality can endanger $\mathrm{PH}$ and $\mathrm{MH}$, and $\mathrm{SC}$ is beneficial to $\mathrm{PH}$ and $\mathrm{MH}$, and there is a mediating effect in the path of IIequality on $\mathrm{PH}$ and $\mathrm{MH}$. Our new findings demonstrate that there are gender differences, urban-rural differences, and regional differences in the effects of Ilequality and $\mathrm{SC}$ on $\mathrm{PH}$ and $\mathrm{MH}$. These findings can be used as new evidence that Ilequality is harmful to $\mathrm{PH}$ and $\mathrm{MH}$. This study also demonstrates from the two dimensions of cognition and structure that Ilequality can affect $\mathrm{PH}$ and $\mathrm{MH}$ through the effect of various types of $\mathrm{SC}$ and provide a way for developing countries to improve their national $\mathrm{PH}$ and $\mathrm{MH}$. Enlightenment. Future research should further examine the time lag of the impact of Ilequality on $\mathrm{SC}$ and $\mathrm{PH}$ and $\mathrm{MH}$.

\section{Abbreviations}

CFPS: China Family Panel Studies Database; COA: Coefficient of action; PDV: Province dummy variable; CV: Control variable; DV: Dependent variable; IV: Independent variable; ICV: Individual characteristic variables; SCV: Social 
characteristic variables; Kl: Kakwani index; MH: Mental health; PH: Physical health; SC: Social capital; llequality: Income inequality; ME: Medical environment; OR: Odds Ratio; TE: Total effect; DE: Direct effect; IE: Intermediary effect.

\section{Supplementary Information}

The online version contains supplementary material available at https://doi. org/10.1186/s12939-021-01560-w.

Additional file 1: Table S1. Multicollinearity Test Results. Table S2. Regression results of the impact of llequality and SC on MH. Table S3. Regression results of the impact of llequality and SC on PH. Table S4. Summary results of robustness analysis. Table S5. Test results of gender and household registration heterogeneity. Table S6. Results of regional heterogeneity test.

\section{Acknowledgements}

The research data comes from the China Family Panel Studies Database (CFPS) of the Institute of Social of Peking University (ISSS), which reflects the changes in Chinese society, economy, population, education, and health, and provides a data basis for this research. Professor Lulin Zhou and the team members of the Medical Insurance Research Center of Jiangsu University for their helpful comments on the research ideas. Finally, I would like to thank the reviewers of the article for repeatedly revising the article and putting forward valuable suggestions for revisions.

\section{Authors' contributions}

Study design, data cleansing, statistical analysis, Yuanyuan He and Lvlin Zhou; supervision, Lvlin Zhou and Jun Wu; writing —original draft, Lvlin Zhou and Junshan Li; writing — review editing, Yuanyuan He, Junshan Li; financial support, Lvlin Zhou. The author(s) read and approved the final manuscript.

\section{Funding}

This study was supported in parts by The National Natural Science Foundation of China General Program (71974064) Value-oriented chronic disease outpatient insurance payment model construction and support strategy research; National Social Science Foundation later funded project (19FGLB006) Maternity insurance and employee basic medical insurance combined implementation issues research; 2020 province The Research and Practice Innovation Plan for Graduate Students (KYCX20_3053) Research on the Response Strategies of Medical Security Participating in the Management of Public Health Emergencies.

\section{Availability of data and materials}

The data that support the findings of this study are available from the Institute of Social of Peking University (ISSS). Data and any supplementary material related to this article can be obtained from the correspondence author on request.

\section{Declarations}

Ethics approval and consent to participate

No human subject was involved in this study.

\section{Consent for publication}

Not applicable.

\section{Competing interests}

All the authors declare that there were no conflicts of interest.

\section{Author details}

${ }^{1}$ School of Management, Jiangsu University, Zhenjiang 212002, Jiangsu, China. ${ }^{2}$ School of Economics and Management, Jiangxi University of Chinese Medicine, Nanchang 330000, Jiangxi, China.

Received: 18 May 2021 Accepted: 25 September 2021

Published online: 06 November 2021

\section{References}

1. Benjamin $D$, Brandt $L$, Giles J. Did higher inequality impede growth in rural China? Econ J. 2011;121(557):1281-309. https://doi.org/10.1016/ S0140-6736(17)30571-8.

2. Bor J, Cohen $\mathrm{GH}$, Galea S. Population health in an era of rising income inequality: USA, 1980-2015. Lancet. 2017;389(10077):1475-90. https:// doi.org/10.1016/50140-6736(17)30571-8.

3. Ravallion M. Income inequality in the developing world. Science. 2014:344(6186):851-5. https://doi.org/10.1126/science.1251875.

4. Luo W, Xie Y. Economic growth, income inequality and life expectancy in China. Soc Sci Med. 2020;256:113046. https://doi.org/10.1016/j.socsC imed.2020.113046.

5. Pickett KE, Wilkinson RG. Income inequality and health: a causal review. Soc Sci Med. 2015;128:316-26. https://doi.org/10.1016/j.socscimed.2014. 12.031.

6. Gunasekara Fl, Carter KN, Crampton P, Blakely T. Income and individual deprivation as predictors of health over time. Int J Public Health. 2013;58(4):501-11. https://doi.org/10.1007/s00038-013-0450-9.

7. Kawachi I, Kennedy BP. The relationship of income inequality to mortality: does the choice of indicator matter? Soc Sci Med. 1997:45(7):1121-7. https://doi.org/10.1016/S0277-9536(97)00044-0.

8. Wilson M, Daly M. Life expectancy, economic inequality, homicide, and reproductive timing in Chicago neighbourhoods. BMJ. 1997;314(7089):1271. https://doi.org/10.1136/bmj.314.7089.1271.

9. Shmueli A. Income-related inequalities in health and health services use in Israel. Isr J Health Policy Res. 2014;3(1):1-8. https://doi.org/10.1186/ 2045-4015-3-37.

10. Cuesta MB, Budría S. Income deprivation and mental well-being: the role of non-cognitive skills. Econ Hum Biol. 2015;17:16-28. https://doi.org/10. 1016/j.ehb.2014.11.004

11. Aida J, Kondo K, Kondo N, Watt RG, Sheiham A, Tsakos G. Income inequality, social capital and self-rated health and dental status in older Japanese. Soc Sci Med. 2011;73(10):1561-8. https://doi.org/10.1016/j. socscimed.2011.09.005.

12. Kim KT. Revisiting the income inequality hypothesis with 292 oecd regional units. Int J Health Serv. 2019;49(2):360-70. https://doi.org/10. 1177/0020731418814105.

13. Silbersdorff A, Lynch J, Klasen S, Kneib T. Reconsidering the incomehealth relationship using distributional regression. Health Econ. 2018;27(7):1074-88. https://doi.org/10.1002/hec.3656.

14. Alwadi M, Vettore MV. Contextual income inequality and adolescents' oral-health-related quality of life: a multi-level analysis. Int Dent J. 2019;69(3):463-71. https://doi.org/10.1111/idj.12504.

15. Karlsson M, Nilsson T, Lyttkens $\mathrm{CH}$, Leeson $\mathrm{G}$. Income inequality and health: Importance of a cross-country perspective. Soc Sci Med. 2010;70(6):875-85. https://doi.org/10.1016/j.socscimed.2009.10.056.

16. Zhang $T, X u Y$, Ren J, Sun L, Liu C. Inequality in the distribution of health resources and health services in china: hospitals versus primary care institutions. Int J Equity Health. 2017;16(14):2-8. https://doi.org/10.1186/ s12939-017-0543-9.

17. Liu B, Wei YD, Simon CA. Social capital, race, and income inequality in the United States. Sustainability. 2017;9(2):248-61. https://doi.org/10.3390/ su9020248.

18. Nieminen T, Härkänen T, Martelin T, Borodulin K, Koskinen S. Social capital and all-cause mortality among Finnish men and women aged 30-79. Eur J Public Health. 2015;25(6):972-8. https://doi.org/10.1093/eurpub/ ckv058.

19. Noguchi M, Kobayashi T, Iwase T, Suzuki E, Kawachi I, Takao S. Social capital and suicidal ideation in community-dwelling older residents: a multilevel analysis of 10, 094 subjects in Japan. Am J Geriatr Psychiatry. 2017;25(1):37-47. https://doi.org/10.1016/j.jagp.2016.10.014.

20. Choi KW, Stein MB, Nishimi KM, Ge T, Coleman JR, Chen CY, et al. An exposure-wide and Mendelian randomization approach to identifying modifiable factors for the prevention of depression. Am J Psychiatry. 2020;177(10):944-54. https://doi.org/10.1176/appi.ajp.2020.19111158.

21. Zhao GC, Gu H, Guo JF. Changes in socioeconomic status and inequality in utilization of medical services: 2000-2011. J Public Adm. 2016;13(02):107-118+158. https://doi.org/10.16149/j.cnki.23-1523.2016. 02.010 [in Chinese] 
22. Xu XL, Zhou LL, Chen X. The heterogeneity of social capital and health statusof land-losing farmers under the background of urbanization. China Rural Observ. 2017;05:74-86 [in Chinese].

23. Kondo N. Socioeconomic disparities and health: impacts and pathways. J Epidemiol. 2012;22(1):2-6. https://doi.org/10.2188/jea.JE20110116.

24. Vilhjalmsdottir A, Gardarsdottir RB, Bernburg JG, Sigfusdottir ID. Neighborhood income inequality, social capital and emotional distress among adolescents: a population-based study. J Adolesc. 2016;51:92-102. https://doi.org/10.1016/j.adolescence.2016.06.004.

25. Varbanova $\vee$, Beutels $P$. Recent quantitative research on determinants of health in high income countries: a scoping review. PLoS One. 2020;15(9):e0239031. https://doi.org/10.1371/journal.pone.0239031.

26. Kohler U, Karlson KB, Holm A. Comparing coefficients of nested nonlinear probability models. Stata J. 2011;11(3):420-38. https://doi.org/10.1007/ s00355-010-0469-2.

27. Karlson KB, Breen R, Holm A. Total, direct, and indirect effects in logit and probit models. Sociol Methods Res. 2013;42:164-91. https://doi.org/10. 1177/0049124113494572.

28. Smith EK, Lacy MG, Mayer A, et al. Performance simulations for categorical mediation: analyzing khb estimates of mediation in ordinal regression models. Stata J. 2019;19(4):913-30. https://doi.org/10.1177/1536867X19 893638.

29. Yang J, Deng DS, Shen Y. Human capital, social security and income inequality of Chinese residents: based on the perspective of individual relative deprivation. Insur Res. 2019;06(009):111-24. https://doi.org/10. 13497/j.cnki.is.2019.06.009 [in Chinese].

30. Yitzhaki S. Relative deprivation and the gini coefficient: reply. Q J Econ. 1980;95(3):575-6. https://doi.org/10.2307/1885095.

31. Kakwani N. The relative deprivation curve and its applications. J Bus Econ Stat. 1984;2(4):384-94. https://doi.org/10.1080/07350015.1984.10509412.

32. Podder N. Relative deprivation, envy and economic inequality: reply. Kyklos. 2010;52(3):449-52. https://doi.org/10.1111/j.1467-6435.1999. tb00227.x.

33. Adjaye-Gbewonyo K, Kawachi I. Use of the Yitzhaki Index as a test of relative deprivation for health outcomes: a review of recent literature. Soc Sci Med. 2012;75(1):129-37. https://doi.org/10.1016/j.socscimed.2012.03.004.

34. Putnam RD. Tuning in, tuning out: the strange disappearance of social capital in America. PS Polit Sci Polit. 1995;28(4):664-83. https://doi.org/10. 1017/S1049096500058856.

35. Moore S, Kawachi I. Twenty years of social capital and health research: a glossary. J Epidemiol Community Health. 2017;71(5):513-7. https://doi. org/10.1136/jech-2016-208313.

36. Dong J, Zhao C. Research on the influence of family support on farmers' entrepreneurship motivation - also on the substitute role of human relationship. China Popul Sci. 2019;(01):61-75+127 https://doi.org/CNKl: SUN:ZKRK.0.2019-01-006. [in Chinese].

37. Yang RD, Chen BK, Zhu SE. A study on the demand behavior of rural households' private loan based on the perspective of social networks. Econ Res. 2011;46(11):116-29 https://doi.org/ CNKI:SUN:JJYJ.0.2011-11-011. [in Chinese].

38. Yang J, Ding SJ, Deng DS. Research on the impact of human capital and social capital on the income inequality of landless farmers. China Popul Resour Environ. 2019;29(03):148-58 https://doi.org/ CNKI:SUN:ZGRZ.0.2019-03-016. [in Chinese].

39. Wang SH, Hu ZL. An empirical study on the impact of social networks and household debt on income gap. Econ Surv. 2020;37(05):19-25. https:// doi.org/10.15931/j.cnki.1006-1096.20200724.010 [in Chinese].

40. Su ZP, Zhang YL. The impact of income inequality on the health of rural residents: verification based on the microscopic perspective of relative deprivation. Agric Technol Econ. 2021;07(03):132-44. https://doi.org/10. 13246/j.cnki.jae.2021.03.010 [in Chinese].

41. Yang J, Sun F, Shen Y. Will income inequality deprive farmers of happiness? An analysis based on the adjustment effect of social capital. J Shanxi Univ Finance Econ. 2019;41(07):1-13. https://doi.org/10.13781/j. cnki.1007-9556.2019.07.001 [in Chinese].

42. Feng K, Wu JY. Income gap and health equity: an empirical study based on CFPS data in 2018. Finance Econ. 2020;10:121-32 [in Chinese].

43. Jaumotte F, Lall S, Papageorgiou C. Rising income inequality: technology, or trade and financial globalization? IMF Econ Rev. 2013;61(2):271-309. https://doi.org/10.1057/imfer.2013.7.

44. Mackenbach JD, Lakerveld J, Oostveen YV, et al. The mediating role of social capital in the association between neighbourhood income inequality and body mass index. Eur J Public Health. 2017;27(2):218-23. https://doi.org/10.1093/eurpub/ckw157.

45. Taube E, Kristensson J, Sandberg M, Midlöv P, Jakobsson U. Loneliness and health care consumption among older people. Scand J Caring Sci. 2015;29(3):435-43. https://doi.org/10.1111/scs.12147.

46. Yiengprugsawan V, Welsh J, Kendig H. Social capital dynamics and health in mid to later life: Findings from Australia. Qual Life Res. 2018;27(5):127782. https://doi.org/10.1007/s11136-017-1655-9.

47. Vanderweele T, Vansteelandt S. Mediation analysis with multiple mediators. Epidemiol Methods. 2014;2(1):95-115. https://doi.org/10.1515/ em-2012-0010.

48. Mira RS, Marcenes W, Stansfeld SA, Bernabé E. Cumulative socio-economic disadvantage and traumatic dental injuries during adolescence. Dental Traumatol. 2021;37(2):338-44. https://doi.org/10.1111/edt.12634.

49. Lu YC, Walker R, Richard P, Younis M. Inequalities in poverty and income between single mothers and fathers. Int J Environ Res Public Health. 2020;17(1):135. https://doi.org/10.3390/ijerph17010135.

50. Lv ZK, Zeng $X$. The impact of urban and rural income inequality on consumption structure-Based on the perspective of bounded rational decision-making. J Xiangtan Univ (Philos Soc Sci Ed). 2020;44(06):74-9. https://doi.org/10.13715/j.cnki.jxupss.2020.06.011 [in Chinese].

51. Li Y, LV GM. Research on the sources and channels of inequality of opportunity in China. China Ind Econ. 2019;(09):60-78. https://doi.org/10. 19581/j.cnki.ciejournal.2019.09.004 [in Chinese].

52. Wang M, Yang Y. Complexity and bias in cross-sectional data with binary disease outcome in observational studies. Stat Med. 2020;40(4):950-62. https://doi.org/10.1002/sim.8812.

53. Tetzlaff F, Epping J, Sperlich S, Tetzlaff J. Widening income inequalities in life expectancy? Analysing time trends based on German health insurance data. J Epidemiol Community Health. 2020;74(7):592-7. https://doi. org/10.1136/jech-2019-212966.

54. Gadalla TM, Fuller-Thomson E. Examining the lag time between statelevel income inequality and individual disabilities: a multilevel analysis. Am J Public Health. 2008;98(12):2187-90. https://doi.org/10.2105/AJPH. 2008.134940.

55. Hu AN. Subjective variables explain subjective variables: methodological analysis. Society. 2019;39(3):183-209. https://doi.org/10.15992/j.cnki.311123/c.2019.03.006 [in Chinese].

\section{Publisher's Note}

Springer Nature remains neutral with regard to jurisdictional claims in published maps and institutional affiliations. 